\title{
Experienced Homophobia and HIV Infection Risk Among U.S. Gay, Bisexual, and Other Men Who Have Sex with Men: A Meta-Analysis
}

\author{
William L. Jeffries IV, PhD, ${ }^{1}$ Stephen A. Flores, PhD, ${ }^{1}$ Cherie R. Rooks-Peck, PhD, ${ }^{1}$ Deborah J. Gelaude, MA, \\ Lisa Belcher, PhD, ${ }^{1}$ Philip M. Ricks, PhD, ${ }^{2}$ and Gregorio A. Millett, $\mathrm{MPH}^{3}$
}

\begin{abstract}
Purpose: Experienced homophobia-negative treatment and perceptions that gay, bisexual, and other men who have sex with men (MSM) encounter because of their sexual orientations-may promote HIV infection among MSM. We conducted a rapid review and meta-analysis to examine experienced homophobia in relation to HIV infection risk.

Methods: We searched Embase, MEDLINE, PsycINFO, and Sociological Abstracts to acquire data from U.S. studies published during 1992-2017. Studies examined experienced homophobia in relation to sexual risk behavior, poor HIV care continuum engagement, and diagnosed HIV infection. Random-effects models yielded summary odds ratios (ORs) and 95\% confidence intervals (CIs).

Results: Experienced homophobia was associated with having any sexual risk behavior $(\mathrm{OR}=1.33,95 \% \mathrm{CI}=1.25-$ $\left.1.42, I^{2}=89.2 \%\right)$, receptive condomless anal sex $(\mathrm{CAS})\left(\mathrm{OR}=1.33,95 \% \mathrm{CI}=1.14-1.56, I^{2}=63.6 \%\right)$, HIVdiscordant CAS $\left(\mathrm{OR}=1.66,95 \% \mathrm{CI}=1.29-2.13, I^{2}=85.3 \%\right)$, an increased number of sex partners $(\mathrm{OR}=1.16$, $\left.95 \% \mathrm{CI}=1.13-1.19, I^{2}=0.0 \%\right)$, diagnosed HIV infection $\left(\mathrm{OR}=1.34,95 \% \mathrm{CI}=1.10-1.64, I^{2}=86.3 \%\right)$, and poor HIV care continuum engagement among MSM living with $\mathrm{HIV}\left(\mathrm{OR}=1.45,95 \% \mathrm{CI}=1.02-2.08, I^{2}=47.0 \%\right)$. Effect sizes for any sexual risk behavior were larger in samples with $\geq 50 \%$ Black or Latino (vs. White) MSM and for family-based mistreatment and perceived sexual minority stigma (vs. other homophobia types).

Conclusion: Experienced homophobia is associated with HIV infection risk among MSM. Its association with sexual risk behavior may be stronger among Black and Latino (vs. White) MSM and for family-based mistreatment and perceived sexual minority stigma (vs. other homophobia types). Research is needed to better understand causality in these relationships and the role of interventions to reduce homophobia.
\end{abstract}

Keywords: bisexual, gay, homophobia, meta-analysis, social determinants of health, stigma

\section{Introduction}

$\mathbf{T}$ HE IMPACT OF HIV on gay, bisexual, and other men who have sex with men (MSM) warrants an examination of its underlying causes. MSM account for $2 \%$ of the U.S. population, but they accounted for $69.9 \%$ of new HIV diagnoses in $2017 .{ }^{1}$ MSM are 87.9 times as likely as behaviorally heterosexual men to ever be diagnosed with HIV infection. ${ }^{2}$ During 2012-2017, when new HIV diagnoses decreased $21.6 \%$ among behaviorally heterosexual men, diagnoses remained stable among MSM ( 26,000 new diagnoses annually). ${ }^{1}$
Black, Latino, and young (aged $\leq 29$ years) MSM were most disproportionately affected. HIV interventions for MSM have historically focused on individual-level biological and behavioral determinants of HIV infection (e.g., sexually transmitted infections and condomless anal sex [CAS], respectively). ${ }^{3}$ Individual-level behavior remains the focus of recent interventions that promote adherence to antiretroviral therapy and pre-exposure prophylaxis. ${ }^{4}$

Fundamental cause theory offers a critique to individuallevel prevention approaches. The theory argues that social conditions, not individual-level factors, are the underlying

\footnotetext{
${ }^{1}$ Division of HIV/AIDS Prevention, National Center for HIV/AIDS, Viral Hepatitis, STD, and TB Prevention, Centers for Disease Control and Prevention, Atlanta, Georgia, USA.

${ }^{2}$ Division of Global Health Protection, Center for Global Health, Centers for Disease Control and Prevention, Atlanta, Georgia, USA.

${ }^{3}$ amfAR, The Foundation for AIDS Research, Washington, District of Columbia, USA.

Abstract presentation: A poster presentation containing some of this article's findings occurred at the 22nd International AIDS Conference in Amsterdam, the Netherlands, July 24, 2018.
} 
causes of disease disparities that exist among privileged and marginalized groups (e.g., heterosexual persons and MSM, respectively). ${ }^{5}$ The reason is that the latter, due to their marginalized social status, have life experiences that inhibit access to health-protective resources, ${ }^{6}$ promote unhealthy behaviors (e.g., substance use) as coping mechanisms, ${ }^{6}$ and inhibit societal responses to health problems that affect them. ${ }^{7}$

Fundamental cause theory is useful for understanding experienced homophobia in relation to HIV infection risk. Psychologist George Weinberg originally introduced "homophobia" in 1972 to denote "fear of homosexuals," 8 but nowadays, it connotes society's negative treatment and perceptions of sexual minority people, including $\mathrm{MSM}^{7}$ Discrimination, harassment, and violence are notable aspects of experienced homophobia. ${ }^{9}$ Like racism and other forms of oppression, homophobia stems from "structural stigma" within societies, ${ }^{10}$ but often manifests interpersonally via mistreatment from others. ${ }^{9}$ Its varied manifestations have led researchers to adopt different terms to describe experienced homophobia and social phenomena contributing to it. Examples include "structural stigma,"10 "enacted stigma," "11 "minority stress," 12 and "experiences of homophobia." ${ }^{13}$ Some researchers use broader terms-for example, "heterosexism" and "sexual stigma" and "perceived sexual minority stigma" - that encapsulate all antigay experiences and perceptions. ${ }^{9,14,15}$ In this article, we use "experienced homophobia" to summarily describe these varied terms, which ultimately point to the negative treatment that MSM encounter because of their sexual orientations.

Experienced homophobia deserves closer attention in HIV research. First, HIV diagnosis rates remain persistently high among MSM despite the remarkably large U.S. investment for domestic HIV care, prevention, and research. During 2013-2017, this investment increased from 22 billion to 26 billion USD annually, and most of this money was earmarked for MSM. ${ }^{16}$ This suggests that factors other than individual-level factors addressed in current prevention efforts underlie HIV's disproportionate impact among MSM. Second, populationbased prospective studies, including studies using experimental designs, ${ }^{17}$ show that "structural stigma" (one form of experienced homophobia) is predictive of needing medical visits for mental health problems ${ }^{17}$ and having psychiatric disorders that can contribute to HIV acquisition. ${ }^{18}$ This suggests that experienced homophobia could similarly promote HIV infection. Third, approximately half of U.S. adults express some level of disapproval of same-sex sexual activity. ${ }^{19}$ This suggests that MSM may routinely experience homophobia and, inevitably, face its unhealthy consequences.

Measurement issues make it difficult to understand experienced homophobia's potential impact on HIV infection risk. Researchers commonly use varied measures because homophobia has different manifestations and exists across multiple societal levels (e.g., interpersonal and structural). Because some forms of experienced homophobia, such as physical violence, are perceptibly more harmful than others, its effect might vary by homophobia type. HIV researchers also use various measures to characterize population-level HIV risk (e.g., condom use or number of sex partners). At times, researchers use "stigma" scales that include items indicative of experienced homophobia. ${ }^{15}$ Consequently, studies have assessed multiple homophobia measures in relation to multiple risk outcomes but lack an overall characterization of this relationship.
Existing studies illustrate this challenge. "Gay-related discrimination,,"20 "experienced homosexual stigma,",21 "harassment,"22 and "violence" 23 have been associated with having diagnosed HIV infection, but comparable measures in other studies have not. ${ }^{1,24,25}$ In many cross-sectional studies, different experienced homophobia measures were associated with CAS, ${ }^{11,26-38}$ high numbers of sex partners, ${ }^{39}$ being in situations that promote vulnerability to CAS (e.g., sex while intoxicated) ${ }^{26}$ and overall "HIV risk. ${ }^{40}$ However, prospective studies of $<80$ MSM in unique subgroups (e.g., caregivers for patients living with AIDS $^{41}$ ) have reported that "sexual minority stress", 42 and "experiences of discrimination" 41 were not associated with CAS. To date, no study has quantitatively summarized the magnitude of experienced homophobia's association with HIV infection risk.

We conducted a meta-analysis to fill this gap. We hypothesized that experienced homophobia is associated with (1) behaviors that increase risk for HIV acquisition and transmission and (2) diagnosed HIV infection. We chose a rapid review to ensure timely synthesis and dissemination of findings due to the need for timely data on MSM. In addition, a meta-analysis was ideal because it estimates effect sizes and notes variation that exists within and across studies. ${ }^{43} \mathrm{We}$ summarized this association with attention to different measures of experienced homophobia and HIV infection risk, yielding implications for research and interventions.

\section{Methods}

\section{Search strategy}

This rapid review followed the Preferred Reporting Items for Systematic Review and Meta-Analysis Protocols statement. ${ }^{44}$ Rapid review methodology allows for the critical appraisal of existing research while limiting particular aspects of the systematic review process, such as a formal quality assessment. ${ }^{45} \mathrm{We}$ searched Embase (OVID), MEDLINE (OVID), PsycINFO (OVID), and Sociological Abstracts (ProQuest) to acquire U.S. studies published during 1992-2017. In MEDLINE and PsycINFO, we cross-referenced medical subject headings (National Library of Medicine) for MSM (i.e., "homosexuality, male"; "male homosexuality"; "bisexuality") and experienced homophobia (i.e., "prejudice"). In all four databases, we simultaneously searched using keywords for MSM (i.e., "men who have sex with men," "homosexual," "bisexual," "gay") and experienced homophobia (i.e., "antigay," "anti-gay," "bullying," "gay-related," "homophobia," "homonegative," "stigma," "oppression," "homeless," and "prejudice"). This broad approach facilitated the inclusion of all studies that might have examined experienced homophobia without referring to it as such (e.g., studies of HIV risk among homeless young men forced to leave home for being gay). We developed this search strategy for a larger study examining experienced homophobia in relation to multiple health outcomes and behaviors. Therefore, we did not include search terms specific to HIV.

\section{Inclusion criteria}

Studies included in our analysis had to (1) provide quantitative data describing associations between at least one type of experienced homophobia and at least one health outcome or behavior among MSM; (2) use samples of U.S. residents; 
(3) be published in English; and (4) appear in peer-reviewed journals. Due to the nature of our study, we included any study design that provided an association between homophobia and HIV infection risk. We excluded studies that were confounded by any ongoing HIV intervention.

\section{Data abstraction}

To ensure a timely screening process, one author reviewed citations and full-text articles identified by the search at the title and abstract levels, followed by the full report. Six members of our research team used a standardized abstraction form to record characteristics and outcomes of studies that met inclusion criteria. Three 2-person teams separately examined one third of the studies. Team members independently abstracted data and then compared abstractions with their partners. Teams discussed and reconciled any coding differences.

We established criteria to standardize the types of data that we abstracted. Bivariate associations were preferred as they provide the most comparable effects across studies. When study authors only provided data from multivariable models, we selected data from the model with the fewest number of covariates. When multiple studies used the same database to provide data for a given outcome, we abstracted data from only the most recent or generalizable study. When authors reported different outcomes from the same database across multiple reports, we ab- stracted all effect sizes. We abstracted effect size data reported for the entire sample as well as relevant subgroups. This required combining data at the study level when authors only reported by subgroup. We contacted authors to obtain quantitative data when authors only narratively described associations or provided inadequate quantitative data for effect size computations.

\section{Experienced homophobia variables}

All studies measured experienced homophobia as negative treatment that MSM encountered because of their actual or perceived sexual orientation. In this meta-analysis, we included an overall variable for experienced homophobia and specific types of homophobia that studies assessed. These types included discrimination (e.g., unfair treatment), verbal harassment (e.g., name calling), family-based mistreatment (e.g., forced housing displacement), physical violence (e.g., being beaten up), and gay-related stress. We also included sexual minority stigma, both perceived (e.g., believing most people in one's community were homophobic) and structural (e.g., county-level proportion of residents who attended non-gayfriendly places of worship). We included the latter because these forms of stigma are precursors, markers, and sequelae of homophobic experiences and are, therefore, important to consider when examining aspects of experienced homophobia that are difficult to measure directly. ${ }^{9,10}$
Records excluded

$\mathrm{N}=3,235$
FIG. 1. Flow diagram of the study selection process. 


\section{HIV infection risk variables}

The outcomes of interest included recent (within the past year) CAS, sex under the influence of drugs or alcohol, number of sex partners, and risky sexual situations-which we combined into an index of sexual risk behavior-having diagnosed HIV infection, ever having a diagnosed sexually transmitted infection, and HIV status disclosure (among MSM living with HIV). We also examined nonadherence to antiretroviral therapy, not being retained in HIV care, and having a detectable viral load. However, because we acquired only one effect size for each of these outcomes, we combined these data into a summary outcome indicating poor HIV care continuum engagement.

\section{Effect size calculation and analytic approach}

We used standard formulas to transform abstracted data into $\log$ odds, which we weighted by the inverse variance and aggregated using the random-effects model. ${ }^{43}$ The summary effect estimate was calculated using Comprehensive Meta-Analysis Version 2 (Biostat, Englewood, New Jersey). Effects were transformed into odds ratios (ORs) for interpretation. In instances that data in one study needed to be combined (e.g., authors only reported effects separately by race/ethnicity or across homophobia measures or outcomes), we used the fixed-effects model to meta-analytically combine those effect sizes to generate an effect size for our analysis. Regardless of combinations, each meta-analysis could only contain one effect per study. We calculated $I^{2}$ to determine the percentage of effect size variability caused by heterogeneity across studies. ${ }^{46} I^{2}$ values of $25 \%, 50 \%$, and $75 \%$ indicated low, medium, and high heterogeneity, respectively.

Next, subgroup analyses examined whether summary effect estimates for sexual risk behavior, sex under the influence of drugs or alcohol, number of sex partners, or having diagnosed HIV infection differed by race/ethnicity or age. Effect estimates from studies that contained samples of $\geq 50 \%$ Black, Latino, or White MSM were aggregated to obtain summary effect estimates. To determine the effects for young MSM, we aggregated effect estimates from studies that contained $\geq 50 \%$ MSM aged $\leq 29$ years for each outcome. Summary effect estimates were also calculated for the association between different types of experienced homophobia (e.g., discrimination, physical violence, verbal harassment) and sexual risk behavior, CAS, and having diagnosed HIV infection.

Finally, we assessed publication bias by visual inspection of funnel plot asymmetry ${ }^{47}$ and validated it by Begg and Mazumdar's rank correlation. ${ }^{48}$ In addition, the randomeffects fail-safe $N$ was estimated to determine what number of missing studies would be required to nullify the overall mean effect estimate. ${ }^{49}$ Because this study used published secondary data, the Centers for Disease Control and Prevention determined that it was exempt from institutional review board review and approval.

\section{Results}

Our search yielded 3362 references. After removing duplicates, non-U.S. studies, and studies that did not include quantitative data necessary for our meta-analysis, 127 studies met inclusion criteria (Fig. 1). Of these, 44 studies yielded 155 unique effect sizes for HIV-related outcomes. ${ }^{11,13,15,20-42,50-67}$
Some studies used samples in which $\geq 50 \%$ of MSM were Black $(n=10,22.7 \%)$, Latino $(n=14,31.8 \%)$, White $(n=9$, $20.5 \%)$, or young $(n=14,31.8 \%)$. Nearly all studies used cross-sectional designs $(n=42,95.5 \%)$. About two fifths $(n=17,38.6 \%)$ recruited MSM residing in multiple U.S. regions, and most $(n=26,59.1 \%)$ included some MSM living with HIV (Table 1).

\section{Meta-analysis}

Figure 2 and Table 2 display summary ORs and 95\% confidence intervals (CIs) for all MSM. Experienced homophobia was associated with having any recent sexual risk behavior $\left(\mathrm{OR}=1.33,95 \% \quad \mathrm{CI}=1.25-1.42, I^{2}=89.2 \%, k=34\right)$, any CAS $\left(\mathrm{OR}=1.21,95 \% \mathrm{CI}=1.13-1.28, I^{2}=84.0 \%, k=22\right)$, receptive CAS $\left(\mathrm{OR}=1.33,95 \% \mathrm{CI}=1.14-1.56, I^{2}=63.6 \%\right.$, $k=6)$, HIV-discordant CAS ( $\mathrm{OR}=1.66,95 \% \mathrm{CI}=1.29-2.13$, $\left.I^{2}=85.3 \%, k=4\right)$, and CAS with casual partners $(\mathrm{OR}=1.58$, $\left.95 \% \mathrm{CI}=1.32-1.87, I^{2}=44.9 \%, k=4\right)$. Experienced homophobia was also associated with having sex under the influence of drugs or alcohol $\left(\mathrm{OR}=1.33,95 \% \mathrm{CI}=1.15-1.54, I^{2}=60.2 \%\right.$, $k=5)$, an increased number of sex partners (OR $=1.16,95 \%$ $\left.\mathrm{CI}=1.13-1.19, I^{2}=0.0 \%, k=5\right)$, diagnosed HIV infection $\left(\mathrm{OR}=1.34,95 \% \mathrm{CI}=1.10-1.64, I^{2}=86.3 \%, k=11\right)$, and poor HIV care continuum engagement among MSM living with HIV $\left(\mathrm{OR}=1.45,95 \% \mathrm{CI}=1.02-2.08, I^{2}=47.0 \%, k=3\right)$. No statistically significant association existed between homophobia and having insertive CAS, being in risky sexual situations, ever having a diagnosed sexually transmitted infection, or HIV status disclosure among MSM living with HIV.

Table 1. Characteristics of InCluded Studies that Measured the Association Between Experienced HOMOPHOBIA AND HIV INFECTION RISK OUTCOMES Among Gay, Bisexual, and Other Men Who Have SeX With Men-United States, 1992-2017

Number of studies (\%)

\section{Race/ethnicity}

$\geq 50 \%$ Black MSM

$10(22.7)$

$\geq 50 \%$ Latino MSM

$14(31.8)$

$\geq 50 \%$ White MSM

$9(20.5)$

No majority racial/ethnic group

Race/ethnicity not reported

$8(18.2)$

Age

$\geq 50 \%$ young MSM

$3(6.8)$

Not $\geq 50 \%$ young MSM

$14(31.8)$

$30(68.2)$

Study design

Cross-sectional

Prospective

$42(95.5)$

$2(4.6)$

U.S. region

Midwest $1(2.3)$

Northeast $10(22.7)$

South 7 (15.9)

West $9(20.5)$

$>1 \quad 17$ (38.6)

Included MSM living with HIV

Yes

$26(59.1)$

No

$18(40.9)$

Total 44 (100)

MSM, gay, bisexual, and other men who have sex with men. 
$\underline{\text { Studies }}$

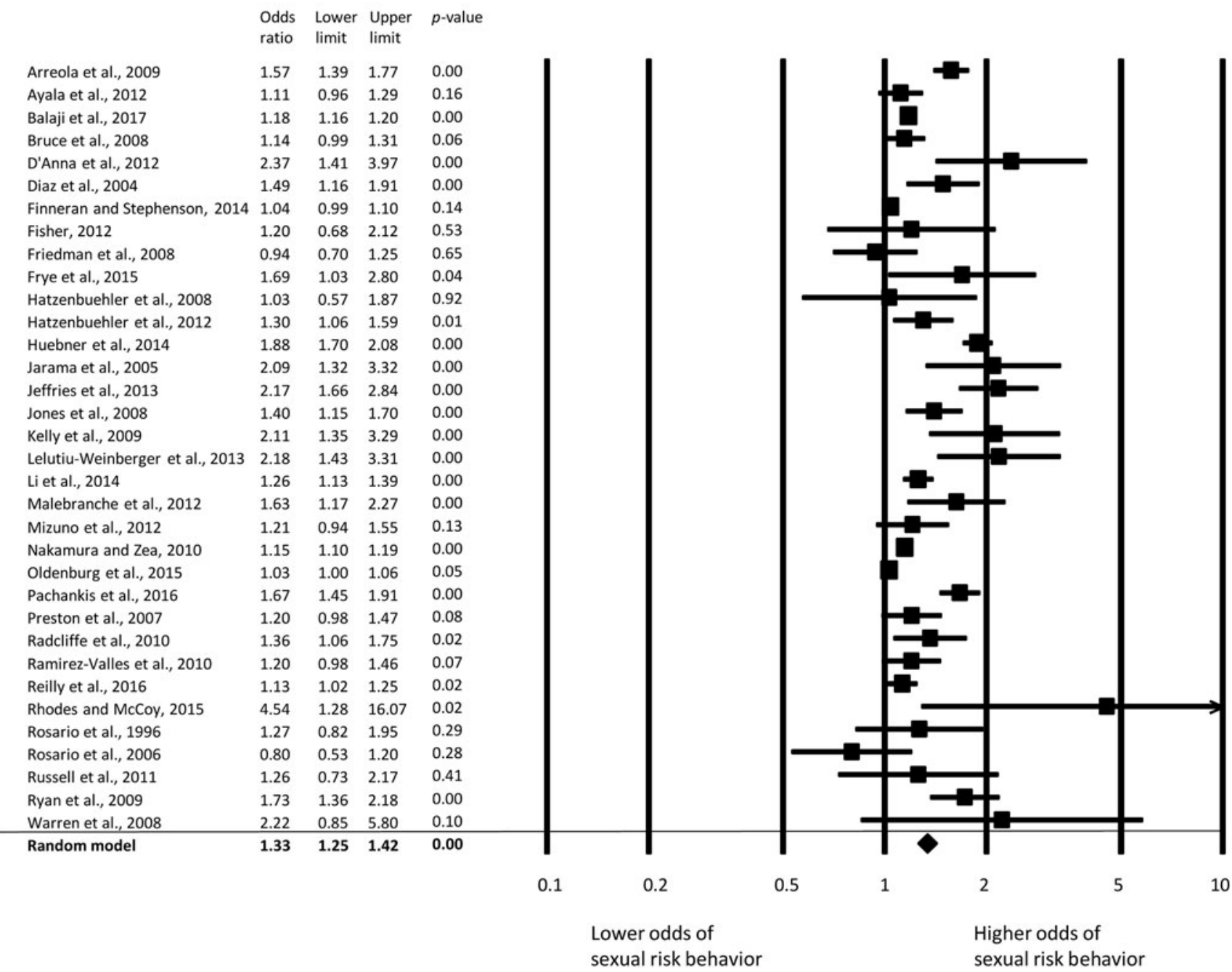

FIG. 2. Forest plot with summary odds ratios for associations between experienced homophobia and any recent sexual risk behavior among gay, bisexual, and other men who have sex with men in the United States, 1992-2017 (N=34).

$I^{2}$ values indicated medium or high heterogeneity in findings across most outcomes. To determine if a specific study accounted for the high heterogeneity, we conducted a sensitivity analysis by assessing the impact of sequentially removing one study at a time from the summary effect estimate. The overall effect estimate remained similar and significant after removal of each study. For the overall sexual risk behavior outcome, we found no evidence of publication bias on the basis of visual inspection of the funnel plot and the Begg test $(Z=0.88 ; p=0.19)$. The fail-safe $N$ was 3555 .

\section{Subgroup analysis}

Table 3 displays summary ORs and 95\% CIs for studies with $\geq 50 \%$ Black, Latino, White, or young MSM. Among studies with $\geq 50 \%$ Black MSM, experienced homophobia was associated with having any recent sexual risk behavior $\left(\mathrm{OR}=1.55,95 \% \mathrm{CI}=1.28-1.88, I^{2}=78.1 \%, k=8\right), \mathrm{CAS}$ $\left(\mathrm{OR}=1.44,95 \% \mathrm{CI}=1.06-1.95, I^{2}=86.4 \%, k=6\right)$, receptive $\mathrm{CAS}\left(\mathrm{OR}=1.44,95 \% \mathrm{CI}=1.09-1.90, I^{2}=0.0 \%, k=2\right)$, and insertive CAS $\left(\mathrm{OR}=1.53,95 \% \mathrm{CI}=1.16-2.00, I^{2}=0.0 \%\right.$, $k=2$ ), but not with diagnosed HIV infection. Among studies with $\geq 50 \%$ Latino MSM, experienced homophobia was associated with having any recent sexual risk behavior $(\mathrm{OR}=1.31$, $\left.95 \% \mathrm{CI}=1.16-1.49, I^{2}=76.6 \%, k=13\right), \mathrm{CAS}(\mathrm{OR}=1.28,95 \%$ $\left.\mathrm{CI}=1.10-1.48, I^{2}=61.7 \%, k=8\right)$, receptive CAS (OR $=1.31$, $\left.95 \% \mathrm{CI}=1.07-1.61, I^{2}=79.7 \%, k=3\right)$, sex under the influence of drugs or alcohol $\left(\mathrm{OR}=1.24,95 \% \mathrm{CI}=1.16-1.32, I^{2}=0 \%\right.$, $k=3)$, and diagnosed HIV infection (OR $=1.72,95 \%$ $\left.\mathrm{CI}=1.42-2.09, I^{2}=0 \%, k=2\right)$, but not with insertive CAS. Among studies with $\geq 50 \%$ White MSM, experienced homophobia was associated with having any recent sexual risk behavior $\left(\mathrm{OR}=1.09,95 \% \mathrm{CI}=1.02-1.18, I^{2}=68.3 \%, k=7\right)$ and CAS $\left(\mathrm{OR}=1.03,95 \% \mathrm{CI}=1.01-1.06, I^{2}=0 \%, k=4\right)$, but not with diagnosed HIV infection. Among studies with $\geq 50 \%$ young MSM, experienced homophobia was associated with having any recent sexual risk behavior $(\mathrm{OR}=1.45,95 \%$ $\mathrm{CI}=1.25-1.68, I^{2}=78.3 \%, k=12$ ), but not with CAS or the number of sex partners.

Table 4 displays summary ORs and 95\% CIs for any recent sexual risk behavior, CAS, and diagnosed HIV infection by the type of homophobia that MSM experienced. Types of 
Table 2. Summary OdDs Ratios for the Association BETWEEN EXPERIENCED HOMOPHOBIA AND HIV InfECTION Risk OUtCOMES AMONG GAy, BiseXuAL, and Other Men Who Have Sex With Men - United States, 1992-2017

\begin{tabular}{|c|c|c|c|}
\hline Measure & $\mathrm{k}$ & $\begin{array}{l}\text { Summary OR } \\
\quad(95 \% \text { CI })\end{array}$ & $\mathrm{I}^{2}$ \\
\hline exual risk behavior, any & 34 & $1.33(1.25-1.42)$ & 89.2 \\
\hline $\mathrm{CAS}, \mathrm{a}$ & 22 & $1.21(1.13-1.28)$ & 84.0 \\
\hline $\mathrm{CAS}, 1$ & 6 & $1.33(1.14-1.56)$ & 63.6 \\
\hline CAS, insertive & 5 & $1.19(0.99-1.43)$ & 53. \\
\hline CAS, HIV discordant & 4 & $1.66(1$. & 85.3 \\
\hline CAS, casual partners & 4 & $1.58(1.3$ & 44.9 \\
\hline $\begin{array}{l}\text { Sex under the influence of } \\
\text { drugs or alcohol }\end{array}$ & 5 & $1.33(1.15-1.54)$ & 60.2 \\
\hline Number of sex partners & 5 & $1.16(1.13-1.19)$ & 0.0 \\
\hline Risky sexual situatio & 3 & $1.88(0.95-3.73)$ & 98.6 \\
\hline Diagnosed HIV infection & 11 & $1.34(1.10-1.64)$ & 86.3 \\
\hline $\begin{array}{l}\text { Diagnosed sexually transmitted } \\
\text { infection, ever }\end{array}$ & 3 & $1.21(0.91-1.60)$ & 40.8 \\
\hline $\begin{array}{l}\text { Poor HIV care continuum } \\
\text { engagement (MSM living } \\
\text { with HIV) }\end{array}$ & 3 & $1.45(1.02-2.08)$ & 47.0 \\
\hline $\begin{array}{l}\text { HIV status disclosure (MSM } \\
\text { living with HIV) }\end{array}$ & 2 & $1.41(0.48-4.21)$ & 88.3 \\
\hline
\end{tabular}

Summary ORs $>1$ indicate greater odds of an outcome among MSM who reported experiencing homophobia compared with MSM who did not report experiencing homophobia.

CAS, condomless anal sex; CI, confidence interval; $k$, number of effect sizes; OR, odds ratio.

homophobia associated with having any recent sexual risk behavior were general experienced homophobia $(\mathrm{OR}=1.36$, $\left.95 \% \mathrm{CI}=1.20-1.54, \quad I^{2}=91.7 \%, \quad k=14\right)$, discrimination $\left(\mathrm{OR}=1.34,95 \% \mathrm{CI}=1.15-1.56, I^{2}=76.6 \%, k=7\right)$, verbal harassment $\left(\mathrm{OR}=1.20,95 \% \mathrm{CI}=1.05-1.40, I^{2}=70.5 \%, k=5\right)$, family-based mistreatment $(\mathrm{OR}=1.68,95 \% \mathrm{CI}=1.40-2.03$, $\left.I^{2}=0.0 \%, k=3\right)$, physical violence $(\mathrm{OR}=1.34,95 \% \mathrm{CI}=1.19$ $\left.1.52, I^{2}=0.0 \%, k=3\right)$, and perceived sexual minority stigma $\left(\mathrm{OR}=1.54,95 \% \mathrm{CI}=1.17-2.04, I^{2}=71.4 \%, k=4\right)$. However, gay-related stress and structural sexual minority stigma were not associated with recent sexual risk behavior (any). Types of homophobia associated with having CAS were general experienced homophobia $(\mathrm{OR}=1.24,95 \% \mathrm{CI}=1.11-1.39$, $\left.I^{2}=82.3 \%, k=10\right)$, discrimination $(\mathrm{OR}=1.36,95 \% \mathrm{CI}=1.15-$ $\left.1.62, I^{2}=76.0 \%, k=7\right)$, and physical violence $(\mathrm{OR}=1.35$, $\left.95 \% \mathrm{CI}=1.13-1.61, I^{2}=0.0 \%, k=2\right)$. However, verbal harassment, family-based mistreatment, and structural sexual minority stigma were not. No specific homophobia types were significantly associated with having diagnosed HIV infection.

\section{Discussion}

Consistent with our first hypothesis, experienced homophobia was associated with behaviors that increase HIV infection risk among MSM: CAS (casual, receptive, and HIV discordant), sex under the influence of drugs or alcohol, an increased number of sex partners, and poor HIV care continuum engagement. Among these findings, it is important to note that receptive CAS is the most risky sexual behavior associated with HIV acquisition, ${ }^{68}$ and HIV-discordant CAS indicates the potential for both HIV acquisition and

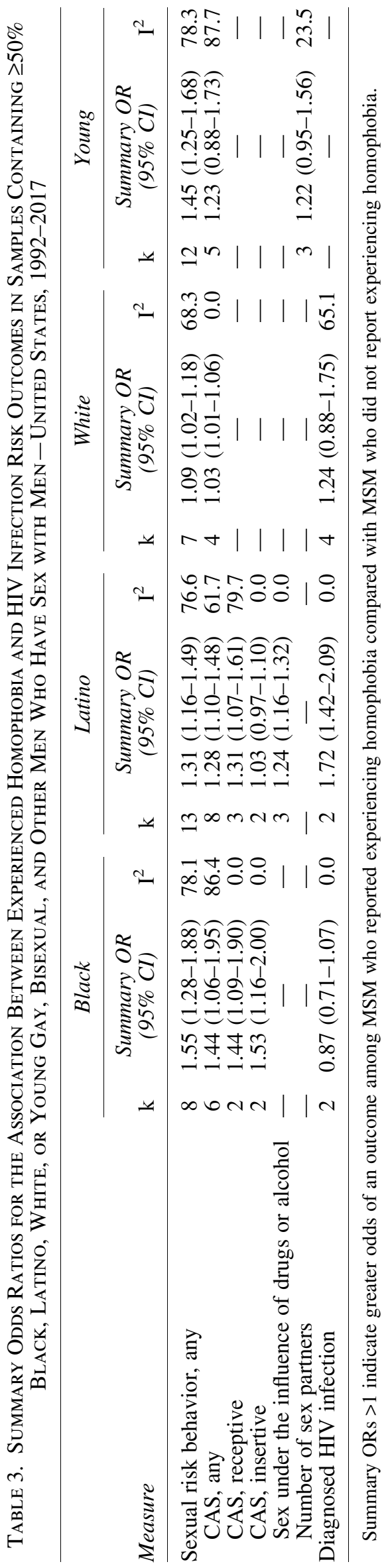


Table 4. Summary Odds Ratios for the Association Between Specific Types of Experienced Homophobia and HIV Infection Risk Outcomes Among Gay, Bisexual, and Other Men Who Have Sex with Men-United States, 1992-2017

\begin{tabular}{|c|c|c|c|c|c|c|c|c|c|}
\hline \multirow[b]{2}{*}{ Homophobia type } & \multicolumn{3}{|c|}{ Sexual risk behavior, any } & \multicolumn{3}{|c|}{$C A S$} & \multicolumn{3}{|c|}{ Diagnosed HIV infection } \\
\hline & $\mathrm{k}$ & $\begin{array}{l}\text { Summary OR } \\
(95 \% \text { CI) }\end{array}$ & $\mathrm{I}^{2}$ & $\mathrm{k}$ & $\begin{array}{l}\text { Summary OR } \\
(95 \% \text { CI) }\end{array}$ & $\mathrm{I}^{2}$ & $\mathrm{k}$ & $\begin{array}{l}\text { Summary OR } \\
(95 \% \text { CI })\end{array}$ & $\mathrm{I}^{2}$ \\
\hline General experienced homophobia & 14 & & 91.7 & 10 & $1.24(1.11-1.39)$ & 82.3 & 4 & $1.29(0.88-1.88)$ & \\
\hline & 7 & & 76.6 & 7 & & & 4 & & \\
\hline nent & 5 & 1.20( & 70.5 & 3 & & 38.1 & 4 & $1.32(0.87-2.01)$ & \\
\hline Fan & 3 & & 0.0 & 3 & & 99.5 & - & - & \\
\hline Physi & 3 & $1.34(1.19-1.52)$ & 0.0 & 2 & $1.35(1.13-1.61)$ & 0.0 & 2 & $1.60(0.64-4.00)$ & \\
\hline Gay-rel & 4 & $1.23(0.83-1.82)$ & 59.6 & - & - & - & - & - & \\
\hline Percei & 4 & $1.54(1.17-2.04)$ & 71.4 & - & - & - & - & - & \\
\hline Structural sexual minority stigma & 3 & $1.26(0.96-1.66)$ & 90.9 & 2 & $1.25(0.84-1.86)$ & 94.3 & - & - & \\
\hline
\end{tabular}

Summary ORs $>1$ indicate greater odds of an outcome among MSM who reported experiencing homophobia compared with MSM who did not report experiencing homophobia.

transmission because it occurs among men with different HIV statuses. Thus, if experienced homophobia increases the risk for these two behaviors, then it would also increase the risk for HIV infection.

Consistent with our second hypothesis, experienced homophobia was associated with having diagnosed HIV infection. We revisit fundamental cause theory to interpret this finding. It is well established that unfavorable social conditions are the underlying reasons that socially marginalized groups experience poorer health than more privileged groups. ${ }^{5}$ In the United States, where heterosexism promotes homophobic attitudes, laws, and policies, it is inevitable that MSM will experience homophobia. ${ }^{9}$ Homophobia limits society's willingness to provide HIV prevention and other health-promoting services to MSM, and it negatively affects society's response to HIV. ${ }^{7}$ Thus, we would expect homophobic experiences, especially when MSM themselves acknowledge and report them, to increase their HIV infection risk. As Stall et al. argued, "[t]he lifelong effects of social marginalization or stigma may ... function in an additive manner to raise levels of high-risk sexual behavior and thus HIV infection itself" (p. 942). ${ }^{69}$

However, it is difficult to ascertain the magnitude of experienced homophobia's effect on HIV infection risk. $I^{2}$ values suggest that heterogeneity across studies generated substantial effect size variability. Different sexual risk behavior measures across the included studies may account for some variability. When we examined specific types of CAS (e.g., insertive and receptive), $I^{2}$ values were lower compared with $I^{2}$ values for any sexual risk behavior, possibly due to the similarity in outcome measures. In stratified analyses, racial/ethnic backgrounds and younger age also appeared to reduce heterogeneity, but to a smaller degree. For sexual risk behavior overall, $I^{2}$ values decreased after stratifying by race/ethnicity and younger age compared with the entire sample. Moreover, we observed reductions in $I^{2}$ for any CAS among Latino and White MSM compared with the entire sample. Fewer studies included in the stratified analysis and similar populations may account for the reduced heterogeneity.

Experienced homophobia's intensity and effect on HIV infection risk likely vary across MSM subgroups. For example, MSM of color (vs. White MSM) are more often challenged by masculine gender roles ${ }^{33}$ family expectations, ${ }^{38}$ and religious norms ${ }^{70}$ that intensify their homophobic experiences. Therefore, MSM of color, who also contend with racism and limited acceptance within White gay communities, ${ }^{29,59}$ likely have fewer psychosocial buffers against homophobia than White MSM. This might explain why effect sizes for any sexual risk behavior and CAS were largest in samples containing mostly Black or Latino (vs. White) MSM.

Different types of experienced homophobia might differentially affect MSM. We found that family-based mistreatment and perceived sexual minority stigma yielded the largest effect sizes. One possible explanation is that familybased mistreatment comes from people whom MSM typically expect to treat them favorably and, therefore, engenders a formidable assault to their personhood. ${ }^{71}$ Similarly, perceived stigma stems from awareness of homophobia emanating from friends and families and, arguably, has a notably strong impact on MSM. ${ }^{32}$ However, our observation that verbal harassment, discrimination, and physical violence were also associated with sexual risk behavior suggests that many manifestations of homophobia affect MSM to some degree. Structural sexual minority stigma was not associated with having sexual risk behavior. This was unexpected given evidence that structural stigma is a robust underlying determinant of health among sexual minority people. ${ }^{10}$

\section{Limitations}

This study has limitations. First, biases within studies that we included may have affected our results. One notable bias involves measurement and conceptualization. Across studies, authors sometimes used the same measure for different types of experienced homophobia or different measures for the same type of experienced homophobia. This highlights the need for better measurement and clearer conceptualization of different types of homophobia. Second, although we used fundamental cause theory as a framework for understanding experienced homophobia's potential effect on HIV infection risk, our findings alone do not establish causality. Third, most studies examined experienced homophobia among "MSM" (not merely gay- or bisexual-identified men), and they did not differentially examine homophobia among MSM of different sexual identities. Consequently, 
our meta-analysis examined experienced homophobia vis-àvis a behavioral dimension of sexual orientation (i.e., selfreported sex with men) rather than sexual identity despite variability in homophobic experiences among MSM of different sexual identities. Finally, we view associations with only two or three effect sizes as preliminary.

\section{Future research}

Future studies could address these limitations and remaining research gaps. More data would help researchers to understand how experienced homophobia potentially affects HIV care continuum engagement at each step of the continuum. Because only one study examined retention in care, antiretroviral therapy adherence, or viral suppression, we had to crudely characterize this relationship using a summary OR. Because we acquired only two effect sizes for diagnosed HIV infection among Black and Latino MSM, and none for young MSM, studies should examine experienced homophobia in relation to this outcome. Furthermore, studies could investigate the relationship between structural sexual minority stigma, which is important for understanding homophobia's impact at the societal level, and HIV infection risk. ${ }^{10}$ Finally, studies using prospective population-based designs would be invaluable for characterizing causal pathways linking experienced homophobia and HIV infection risk.

\section{Conclusion}

As HIV continues its spread among MSM, interventions addressing experienced homophobia are essential. The most widely disseminated interventions for MSM almost exclusively target individual-level risk behaviors rather than the social factors that contribute to these behaviors. ${ }^{72}$ Our findings, alongside other research on experienced homophobia, suggest that interventions should also target society by addressing homophobia. These interventions might reduce HIV diagnoses among MSM by promoting social environments that are conducive to healthy behaviors. ${ }^{71}$ Mass media, social marketing campaigns have considerable promise given their abilities to change community- and society-level attitudes toward MSM. Three of these interventions exist in the United States. ${ }^{73-75}$ Although they have only been implemented in two cities, an evaluation of one of them suggests that it may be efficacious at reducing homophobia if disseminated more widely. ${ }^{75}$ Ultimately, by addressing societal homophobia, MSM can live in health-promoting environments that help to reduce their risk for HIV infection. Devoting greater attention to experienced homophobia in research and interventions could help the United States to achieve its vision of ending the HIV epidemic.

\section{Authors' Contributions}

W.L.J.IV. conceptualized the study, served as its lead writer, and created Figure 1 and Tables 1-4. S.A.F. helped to conceptualize the study, oversaw data management, and analyzed the data. C.R.R.-P. analyzed the data, created Figure 2, and wrote part of the Methods section. D.J.G., L.B., P.M.R., and G.A.M. abstracted the study data. All the authors reviewed the article, provided written comments, and approved the article before submission.

\section{Disclaimer}

The findings and conclusions in this report are those of the authors and do not necessarily represent the official position of the Centers for Disease Control and Prevention.

\section{Acknowledgments}

We are grateful to Julia B. DeLuca, MLIS, Mary M. Mullins, MLIS, and Katherine L. Tucker for assistance with the literature search. Richard J. Wolitski, PhD, and John L. Peterson, PhD, provided helpful thoughts on the conceptualization of this article.

\section{Author Disclosure Statement}

No competing financial interests exist.

\section{Funding Information}

No funding was received for this article.

\section{References}

1. Centers for Disease Control and Prevention: Diagnoses of HIV infection in the United States and dependent areas, 2017. 2018. Available at https://www.cdc.gov/hiv/pdf/ library/reports/surveillance/cdc-hiv-surveillance-report-2017vol-29.pdf Accessed October 9, 2020.

2. Hess KL, Hu X, Lansky A, et al.: Lifetime risk of a diagnosis of HIV infection in the United States. Ann Epidemiol 2017;27:238-243.

3. Herbst JH, Beeker C, Mathew A, et al.: The effectiveness of individual-, group-, and community-level HIV behavioral risk-reduction interventions for adult men who have sex with men: A systematic review. Am J Prev Med 2007;32: S38-S67.

4. Charania MR, Marshall KJ, Lyles CM, et al.: Identification of evidence-based interventions for promoting HIV medication adherence: Findings from a systematic review of U.S.based studies, 1996-2011. AIDS Behav 2014;18:646-660.

5. Phelan JC, Link BG, Tehranifar P: Social conditions as fundamental causes of health inequalities: Theory, evidence, and policy implications. J Health Soc Behav 2010;51 Suppl: S28-S40.

6. Hatzenbuehler ML, Phelan JC, Link BG: Stigma as a fundamental cause of population health inequalities. Am J Public Health 2013;103:813-821.

7. Altman D, Aggleton P, Williams M, et al.: Men who have sex with men: Stigma and discrimination. Lancet 2012; 380:439-445.

8. Weinberg G: Society and the Healthy Homosexual. New York: St. Martin's Press, 1972.

9. Herek GM: Beyond "homophobia": Thinking more clearly about stigma, prejudice, and sexual orientation. Am J Orthopsychiatry 2015;85:S29-S37.

10. Hatzenbuehler ML: Structural stigma: Research evidence and implications for psychological science. Am Psychol 2016;71:742-751.

11. Balaji AB, Bowles KE, Hess KL, et al.: Association between enacted stigma and HIV-related risk behavior among MSM, National HIV Behavioral Surveillance System, 2011. AIDS Behav 2017;21:227-237.

12. Meyer IH: Minority stress and mental health in gay men. J Health Soc Behav 1995;36:38-56. 
13. Diaz RM, Ayala G, Bein E: Sexual risk as an outcome of social oppression: Data from a probability sample of Latino gay men in three U.S. cities. Cultur Divers Ethnic Minor Psychol 2004;10:255-267.

14. Herek GM, McLemore KA: Sexual prejudice. Annu Rev Psychol 2013;64:309-333.

15. Radcliffe J, Doty N, Hawkins LA, et al.: Stigma and sexual health risk in HIV-positive African American young men who have sex with men. AIDS Patient Care STDS 2010; 24:493-499.

16. Kaiser Family Foundation: U.S. federal funding for HIV/ AIDS: Trends over time. 2019. Available at https://www .kff.org/hivaids/fact-sheet/u-s-federal-funding-for-hivaidstrends-over-time/ Accessed October 9, 2020.

17. Hatzenbuehler ML, O'Cleirigh C, Grasso C, et al.: Effect of same-sex marriage laws on health care use and expenditures in sexual minority men: A quasi-natural experiment. Am J Public Health 2012;102:285-291.

18. Hatzenbuehler ML, McLaughlin KA, Keyes KM, Hasin DS: The impact of institutional discrimination on psychiatric disorders in lesbian, gay, and bisexual populations: A prospective study. Am J Public Health 2010;100:452-459.

19. Twenge JM, Sherman RA, Wells BE: Changes in American adults' reported same-sex sexual experiences and attitudes, 1973-2014. Arch Sex Behav 2016;45:1713-1730.

20. Reilly KH, Neaigus A, Jenness SM, et al.: Experiences of discrimination and HIV risk among men who have sex with men in New York City. Am J Mens Health 2016;10: 505-514.

21. Bruce D, Ramirez-Valles J, Campbell RT: Stigmatization, substance use, and sexual risk behavior among Latino gay and bisexual men and transgender persons. J Drug Issues 2008;38:235-260.

22. D'Anna LH, Nguyen HHD, Reynolds GL, et al.: The relationship between sexual minority verbal harassment and utilization of health services: Results from Countywide Risk Assessment Survey (CRAS) 2004. J Gay Lesbian Soc Serv 2012;24:119-139.

23. Huebner DM, Rebchook GM, Kegeles SM: Experiences of harassment, discrimination, and physical violence among young gay and bisexual men. Am J Public Health 2004; 94:1200-1203.

24. Friedman MS, Marshal MP, Stall R, et al.: Gay-related development, early abuse and adult health outcomes among gay males. AIDS Behav 2008;12:891-902.

25. Peterson JL, Bakeman R, Sullivan P, et al.: Social discrimination and resiliency are not associated with differences in prevalent HIV infection in black and white men who have sex with men. J Acquir Immune Defic Syndr 2014;66: 538-543.

26. Arreola SG, Neilands TB, Diaz R: Childhood sexual abuse and the sociocultural context of sexual risk among adult Latino gay and bisexual men. Am J Public Health 2009;99 Suppl 2:S432-S438.

27. Ayala G, Bingham T, Kim J, et al.: Modeling the impact of social discrimination and financial hardship on the sexual risk of HIV among Latino and Black men who have sex with men. Am J Public Health 2012;102 Suppl 2:S242S249.

28. Frye V, Nandi V, Egan J, et al.: Sexual orientation- and race-based discrimination and sexual HIV risk behavior among urban MSM. AIDS Behav 2015;19:257-269.

29. Huebner DM, Kegeles SM, Rebchook GM, et al.: Social oppression, psychological vulnerability, and unprotected inter- course among young Black men who have sex with men. Health Psychol 2014;33:1568-1578.

30. Jeffries WL 4th, Marks G, Lauby J, et al.: Homophobia is associated with sexual behavior that increases risk of acquiring and transmitting HIV infection among black men who have sex with men. AIDS Behav 2013;17:1442-1453.

31. Kelly BC, Bimbi DS, Izienicki H, Parsons JT: Stress and coping among HIV-positive barebackers. AIDS Behav 2009;13:792-797.

32. Lelutiu-Weinberger C, Pachankis JE, Golub SA, et al.: Age cohort differences in the effects of gay-related stigma, anxiety and identification with the gay community on sexual risk and substance use. AIDS Behav 2013;17:340-349.

33. Malebranche DJ, Gvetadze R, Millett GA, Sutton MY: The relationship between gender role conflict and condom use among black MSM. AIDS Behav 2012;16:2051-2061.

34. Nakamura N, Zea MC: Experiences of homonegativity and sexual risk behaviour in a sample of Latino gay and bisexual men. Cult Health Sex 2010;12:73-85.

35. Oldenburg CE, Perez-Brumer AG, Hatzenbuehler ML, et al.: State-level structural sexual stigma and HIV prevention in a national online sample of HIV-uninfected men who have sex with men in the United States. AIDS 2015;29:837-845.

36. Pachankis JE, Eldahan AI, Golub SA: New to New York: Ecological and psychological predictors of health among recently arrived young adult gay and bisexual urban migrants. Ann Behav Med 2016;50:692-703.

37. Rhodes SD, McCoy TP: Condom use among immigrant Latino sexual minorities: Multilevel analysis after respondentdriven sampling. AIDS Educ Prev 2015;27:27-43.

38. Ryan C, Huebner D, Diaz RM, Sanchez J: Family rejection as a predictor of negative health outcomes in white and Latino lesbian, gay, and bisexual young adults. Pediatrics 2009; 123:346-352.

39. Hatzenbuehler ML, Pachankis JE, Wolff J: Religious climate and health risk behaviors in sexual minority youths: A population-based study. Am J Public Health 2012;102: 657-663.

40. Russell ST, Ryan C, Toomey RB, et al.: Lesbian, gay, bisexual, and transgender adolescent school victimization: Implications for young adult health and adjustment. J Sch Health 2011;81:223-230.

41. Hatzenbuehler ML, Nolen-Hoeksema S, Erickson SJ: Minority stress predictors of HIV risk behavior, substance use, and depressive symptoms: Results from a prospective study of bereaved gay men. Health Psychol 2008;27:455462.

42. Rosario M, Schrimshaw EW, Hunter J: A model of sexual risk behaviors among young gay and bisexual men: Longitudinal associations of mental health, substance abuse, sexual abuse, and the coming-out process. AIDS Educ Prev 2006;18:444-460.

43. Borenstein M, Hedges LV, Higgins JPT, Rothstein HR: Introduction to Meta-Analysis. Chichester, UK: John Wiley \& Sons, Ltd., 2009.

44. Moher D, Shamseer L, Clarke M, et al.: Preferred reporting items for systematic review and meta-analysis protocols (PRISMA-P) 2015 statement. Syst Rev 2015;4:1.

45. Grant MJ, Booth A: A typology of reviews: An analysis of 14 review types and associated methodologies. Health Info Libr J 2009;26:91-108.

46. Higgins JPT, Thompson SG, Deeks JJ, Altman DG: Measuring inconsistency in meta-analyses. BMJ 2003;327:557560 . 
47. Egger M, Smith GD, Schneider M, Minder C: Bias in metaanalysis detected by a simple, graphical test. BMJ 1997;315: 629-634.

48. Begg CB, Mazumdar M: Operating characteristics of a rank correlation test for publication bias. Biometrics 1994;50: 1088-1101.

49. Rosenberg MS: The file-drawer problem revisited: A general weighted method for calculating fail-safe numbers in meta-analysis. Evolution 2005;59:464-468.

50. Bogart LM, Wagner GJ, Galvan FH, Klein DJ: Longitudinal relationships between antiretroviral treatment adherence and discrimination due to HIV-serostatus, race, and sexual orientation among African-American men with HIV. Ann Behav Med 2010;40:184-190.

51. Finneran C, Stephenson R: Intimate partner violence, minority stress, and sexual risk-taking among U.S. men who have sex with men. J Homosex 2014;61:288-306.

52. Fisher CM: Adapting the Information-motivation-behavioral skills model: Predicting HIV-related sexual risk among sexual minority youth. Health Educ Behav 2012; 39:290-302.

53. Frost DM, Parsons JT, Nanin JE: Stigma, concealment and symptoms of depression as explanations for sexually transmitted infections among gay men. J Health Psychol 2007; 12:636-640.

54. Huebner DM, Davis MC: Perceived antigay discrimination and physical health outcomes. Health Psychol 2007;26:627634.

55. Jarama SL, Kennamer JD, Poppen PJ, et al.: Psychosocial, behavioral, and cultural predictors of sexual risk for HIV infection among Latino men who have sex with men. AIDS Behav 2005;9:513-523.

56. Jones KT, Johnson WD, Wheeler DP, et al.: Nonsupportive peer norms and incarceration as HIV risk correlates for young black men who have sex with men. AIDS Behav 2008;12:41-50.

57. Kass NE, Faden RR, Fox R, Dudley J: Homosexual and bisexual men's perceptions of discrimination in health services. Am J Public Health 1992;82:1277-1279.

58. Li MJ, DiStefano A, Mouttapa M, Gill JK: Bias-motivated bullying and psychosocial problems: Implications for HIV risk behaviors among young men who have sex with men. AIDS Care 2014;26:246-256.

59. Mizuno Y, Borkowf C, Millett GA, et al.: Homophobia and racism experienced by Latino men who have sex with men in the United States: Correlates of exposure and associations with HIV risk behaviors. AIDS Behav 2012;16:724-735.

60. Preston DB, D'Augelli AR, Kassab CD, Starks MT: The relationship of stigma to the sexual risk behavior of rural men who have sex with men. AIDS Educ Prev 2007;19:218-230.

61. Ramirez-Valles J, Kuhns LM, Campbell RT, Diaz RM: Social integration and health: Community involvement, stigmatized identities, and sexual risk in Latino sexual minorities. J Health Soc Behav 2010;51:30-47.

62. Rosario M, Rotheram-Borus MJ, Reid H: Gay-related stress and its correlates among gay and bisexual male adolescents of predominantly Black and Hispanic background. J Community Psychol 1996;24:136-159.

63. Scott HM, Pollack L, Rebchook GM, et al.: Peer social support is associated with recent HIV testing among young black men who have sex with men. AIDS Behav 2014;18: 913-920.

64. Warren JC, Fernandez MI, Harper GW, et al.: Predictors of unprotected sex among young sexually active African American, Hispanic, and White MSM: The importance of ethnicity and culture. AIDS Behav 2008;12:459-468.

65. Wohl AR, Galvan FH, Myers HF, et al.: Do social support, stress, disclosure and stigma influence retention in HIV care for Latino and African American men who have sex with men and women? AIDS Behav 2011;15:1098-1110.

66. Zea MC, Reisen CA, Poppen PJ, et al.: Predictors of disclosure of human immunovirus-positive serostatus among Latino gay men. Cultur Divers Ethnic Minor Psychol 2007; 13:304-312.

67. Zea MC, Reisen CA, Poppen PJ, Díaz RM: Asking and telling: Communication about HIV status among Latino HIVpositive gay men. AIDS Behav 2003;7:143-152.

68. Patel P, Borkowf CB, Brooks JT, et al.: Estimating per-act HIV transmission risk: A systematic review. AIDS 2014; 28:1509-1519.

69. Stall R, Mills TC, Williamson J, et al.: Association of cooccurring psychosocial health problems and increased vulnerability to HIV/AIDS among urban men who have sex with men. Am J Public Health 2003;93:939-942.

70. Jeffries WL 4th, Okeke JO, Gelaude DJ, et al.: An exploration of religion and spirituality among young, HIV-infected gay and bisexual men in the USA. Cult Health Sex 2014;16: 1070-1083.

71. Jeffries WL 4th, Gelaude DJ, Torrone EA, et al.: Unhealthy environments, unhealthy consequences: Experienced homonegativity and HIV infection risk among young men who have sex with men. Glob Public Health 2017;12:116-129.

72. Centers for Disease Control and Prevention: Effective interventions. 2020. Available at https://www.cdc.gov/hiv/ effective-interventions/index.html Accessed August 30, 2020.

73. Frye V, Paige MQ, Gordon S, et al.: Impact of a communitylevel intervention on HIV stigma, homophobia and HIV testing in New York City: Results from project CHHANGE. Stigma Health 2019;4:72-81.

74. Gay Men's Health Crisis: I love my boo. 2020. Available at www.gmhc.org/i-love-my-boo-4 Accessed September 14, 2020.

75. Hull SJ, Davis CR, Hollander G, et al.: Evaluation of the Acceptance Journeys social marketing campaign to reduce homophobia. Am J Public Health 2017;107:173-179.

Address correspondence to:

William L. Jeffries IV, PhD

Division of HIV/AIDS Prevention National Center for HIV/AIDS

Viral Hepatitis, STD, and TB Prevention Centers for Disease Control and Prevention 1600 Clifton Road, MS US8-3 Atlanta, GA 30333

USA

E-mail: wjeffries@cdc.gov 